Article

\title{
Structure and Functions of Topological Metagroups
}

\author{
Sergey V. Ludkowski \\ Department of Applied Mathematics, MIREA-Russian Technological University, av. Vernadsky 78, \\ 119454 Moscow, Russia; sludkowski@mail.ru
}

Received: 5 April 2020; Accepted: 10 June 2020; Published: 14 June 2020

\begin{abstract}
In this article, the structure of topological metagroups was investigated. Relations between topological and algebraic properties of metagroups were scrutinized. A uniform continuity of functions on them was studied. Smashed products of topological metagroups were investigated.
\end{abstract}

Keywords: metagroup; topological; function; smashed product

MSC: 20N05; 22A30; 54C05; 54E15; 54H11

\section{Introduction}

In nonassociative algebra, noncommutative geometry, field theory, and topological algebra there frequently appear binary systems, which are nonassociative generalizations of groups and are related to loops, quasi-groups, and Moufang loops, etc. (see [1-6]). A particular class of quasi-groups known as gyrogroups $G_{y}$ arise as a skew product of a group $A_{y}$ and its automorphism group $A u t\left(A_{y}\right)$. Relations between $(a b) c$ and $a(b c)$ for each $a, b$ and $c$ in $G_{y}$ are provided with the help of the automorphism $\psi$ of $A_{y}$ acting on $c$, where $\psi: G_{y}^{2} \rightarrow A u t\left(A_{y}\right), \psi=\psi_{a, b}$ may depend only on two parameters $a$ and $b$ in $G_{y}$. They found applications in physics for studying Thomas precession with the help of the Lorentz group and its automorphism group [7]. It was investigated and proved in the 20th century that a nontrivial geometry exists if and only if there exists a corresponding unital quasi-group (loop) [2,4].

A very important role in mathematics and quantum field theory play octonions and generalized Cayley-Dickson algebras [8-10]. A multiplicative law of their canonical bases is nonassociative and leads to a more general notion of a metagroup instead of a group [11]. They are used not only in algebra and geometry, but also in noncommutative analysis and PDEs, particle physics, and mathematical physics $[9,10,12-26]$. The preposition meta is used to emphasize that such an algebraic object has properties milder than those of a group. By their axiomatic metagroups are loops (that is, satisfy the conditions of Equations (1)-(3)) with additional weak relations (10). Metagroups were used in [11] to investigate of automorphisms and derivations of nonassociative algebras, as well as cohomologies of nonassociative algebras [27]. Constructions of metagroups from groups or other metagroups with the help of smashed and twisted wreath products, of certain nonassociative algebras and examples were given in $[11,27,28]$.

This article is devoted to the structure of topological metagroups. Specific algebraic and topological features of metagroups are studied in Lemmas 1-4, Theorem 2, and Propositions 1 and 2. Relations between topological and algebraic properties of metagroups are scrutinized. A quotient of a metagroup by its central subgroup is investigated in Theorem 1. A uniform continuity of maps on topological metagroups is studied in Theorem 3 and Corollary 2.

Smashed products of topological metagroups are investigated in Section 3. It is proved in Theorems 4 and 5 and Corollaries 3-5 that there are abundant families of topological metagroups.

All main results of this paper are obtained for the first time. 


\section{Structure of Topological Metagroups}

To avoid misunderstandings, we provide the necessary definition. A reader familiar with $[2,11,27]$ can skip Definition 1.

Definition 1. Let $G$ be a set with a single-valued binary operation (multiplication) $G^{2} \ni(a, b) \mapsto a b \in G$ defined on $G$ satisfying the conditions:

For each $a$ and $b$ in $G$ there is a unique $x \in G$ with

$$
a x=b
$$

and a unique $y \in G$ exists satisfying

$$
y a=b
$$

which are denoted by $x=a \backslash b=\operatorname{Div}_{l}(a, b)$ and $y=b / a=\operatorname{Div}_{r}(a, b)$ correspondingly,

there exists a neutral (i.e., unit) element $e_{G}=e \in G$ :

$$
e g=g e=g
$$

for each $g \in G$.

The set of all elements $h \in G$ commuting and associating with $G$ :

$$
\begin{gathered}
\operatorname{Com}(G):=\{a \in G: \forall b \in G, a b=b a\} \\
N_{l}(G):=\{a \in G: \forall b \in G, \forall c \in G,(a b) c=a(b c)\} \\
N_{m}(G):=\{a \in G: \forall b \in G, \forall c \in G,(b a) c=b(a c)\} \\
N_{r}(G):=\{a \in G: \forall b \in G, \forall c \in G,(b c) a=b(c a)\} \\
N(G):=N_{l}(G) \cap N_{m}(G) \cap N_{r}(G) \\
\mathcal{C}(G):=\operatorname{Com}(G) \cap N(G)
\end{gathered}
$$

is called the center $\mathcal{C}(G)$ of $G$.

We call $G$ a metagroup if a set $G$ possesses a single-valued binary operation and satisfies the conditions of Equations (1)-(3) and

$$
(a b) c=t(a, b, c) a(b c)
$$

for each $a, b$ and $c$ in $G$, where $t(a, b, c)=t_{G}(a, b, c) \in \mathcal{C}(G)$.

Then $G$ is called a central metagroup if in addition to (10) it satisfies the condition:

$$
a b=\mathrm{t}_{2}(a, b) b a
$$

for each $a$ and $b$ in $G$, where $\mathrm{t}_{2}(a, b) \in \mathcal{C}(G)$.

Let $\tau$ be a topology on $G$ such that the multiplication $G^{2} \ni(a, b) \mapsto a b \in G$, the operations $\operatorname{Div}_{l}(a, b)$ and $\operatorname{Div}_{r}(a, b)$ are jointly continuous relative to $\tau$, then $(G, \tau)$ will be called a topological metagroup. Henceforth, it will be assumed that $\tau$ is the $T_{1} \cap T_{3.5}$ topology, maps and functions on metagroups are supposed to be single-valued, if something other will not be specified.

A neighborhood $U$ of the unit element $e$ is called left (right) symmetric, if $U=\operatorname{Inv}_{l}(U)$ (or $U=\operatorname{Inv}_{r}(U)$ respectively), where $\operatorname{Inv}_{l}(a)=\operatorname{Div}_{l}(a, e)$ is a left inversion, $\operatorname{Inv}_{r}(a)=\operatorname{Div}_{r}(a, e)$ is a right inversion. If a neighborhood $U$ is left and right symmetric, then it will be called symmetric.

Elements of a metagroup $G$ will be denoted by small letters, subsets of $G$ will be denoted by capital letters. If $A$ and $B$ are subsets in $G$, then $A-B$ means the difference of them $A-B=\{a \in A: a \notin B\}$. 
Lemma 1. If $G$ is a metagroup, then for each $a$ and $b \in G$ the following identities are fulfilled:

$$
\begin{gathered}
b \backslash e=(e / b) t(e / b, b, b \backslash e) \\
(a \backslash e) b=(a \backslash b) t(e / a, a, a \backslash e) / t(e / a, a, a \backslash b) \\
b(e / a)=(b / a) t(b / a, a, a \backslash e) / t(e / a, a, a \backslash e)
\end{gathered}
$$

Proof. The conditions of Equations (1)-(3) imply that

$$
\begin{gathered}
b(b \backslash a)=a, b \backslash(b a)=a \\
(a / b) b=a,(a b) / b=a
\end{gathered}
$$

for each $a$ and $b$ in $G$. Using Equation (10) and the identities of Equations (15) and (16) we deduce that $e / b=(e / b)(b(b \backslash e))=(b \backslash e) / t(e / b, b, b \backslash e)$

which leads to Equation (12).

Let $c=a \backslash b$, then from the identities of Equations (12) and (15) it follows that

$(a \backslash e) b=(e / a) t(e / a, a, a \backslash e)(a c)$

$=((e / a) a)(a \backslash b) t(e / a, a, a \backslash e) / t(e / a, a, a \backslash b)$,

which provides Equation (13).

Let now $d=b / a$, then the identities of Equations (12) and (16) imply that

$b(e / a)=(d a)(a \backslash e) / t(e / a, a, a \backslash e)=(b / a) t(b / a, a, a \backslash e) / t(e / a, a, a \backslash e)$

which demonstrates (14).

Lemma 2. Assume that $G$ is a metagroup. Then for every $a, a_{1}, a_{2}, a_{3}$ in $G$ and $p_{1}, p_{2}, p_{3}$ in $\mathcal{C}(G)$ :

$$
\begin{gathered}
t\left(p_{1} a_{1}, p_{2} a_{2}, p_{3} a_{3}\right)=t\left(a_{1}, a_{2}, a_{3}\right) \\
t(a, a \backslash e, a) t(a \backslash e, a, e / a)=e
\end{gathered}
$$

Proof. Since $\left(a_{1} a_{2}\right) a_{3}=t\left(a_{1}, a_{2}, a_{3}\right) a_{1}\left(a_{2} a_{3}\right)$ and $t\left(a_{1}, a_{2}, a_{3}\right) \in \mathcal{C}(G)$ for every $a_{1}, a_{2}, a_{3}$ in $G$, then

$$
t\left(a_{1}, a_{2}, a_{3}\right)=\left(\left(a_{1} a_{2}\right) a_{3}\right) /\left(a_{1}\left(a_{2} a_{3}\right)\right)
$$

Therefore, for every $a_{1}, a_{2}, a_{3}$ in $G$ and $p_{1}, p_{2}, p_{3}$ in $\mathcal{C}(G)$ we infer that

$t\left(p_{1} a_{1}, p_{2} a_{2}, p_{3} a_{3}\right)=\left(\left(\left(p_{1} a_{1}\right)\left(p_{2} a_{2}\right)\right)\left(p_{3} a_{3}\right)\right) /\left(\left(p_{1} a_{1}\right)\left(\left(p_{2} a_{2}\right)\left(p_{3} a_{3}\right)\right)\right)$

$=\left(\left(p_{1} p_{2} p_{3}\right)\left(\left(a_{1} a_{2}\right) a_{3}\right)\right) /\left(\left(p_{1} p_{2} p_{3}\right)\left(a_{1}\left(a_{2} a_{3}\right)\right)\right)=\left(\left(a_{1} a_{2}\right) a_{3}\right) /\left(a_{1}\left(a_{2} a_{3}\right)\right)$, since

$$
b /(p a)=p^{-1} b / a, b / p=p \backslash b=b p^{-1}
$$

for each $p \in \mathcal{C}(G)$, $a$ and $b$ in $G$, because $\mathcal{C}(G)$ is the commutative group. Thus $t\left(p_{1} a_{1}, p_{2} a_{2}, p_{3} a_{3}\right)=$ $t\left(a_{1}, a_{2}, a_{3}\right)$.

From the condition of Equation (10), Lemma 1 and the identity in Equation (17) it follows that $t(a, a \backslash e, a)=((a(a \backslash e)) a) /(a((a \backslash e) a))=a /[a t(e / a, a, a \backslash e)]$

$=e / t(a \backslash e, a, e / a)$

for each $a \in G$ implying Equation (18).

Lemma 3. If $(G, \tau)$ is a topological metagroup, then the function $t\left(a_{1}, a_{2}, a_{3}\right)$ is jointly continuous in $a_{1}, a_{2}$, $a_{3}$ in $G$.

Proof. This follows immediately from Equation (19) and Definition 1. 
Lemma 4. Assume that $(G, \tau)$ is a topological metagroup and $U$ is an open subsets in $G$, then for each $b \in G$ the sets $U b$ and bU are open in $G$.

Proof. Take any $c \in U b$ and consider the equation

$$
x b=c
$$

Then from Equation (2) it follows that

$$
x=c / b
$$

Thus $x=\psi_{b}(c)$, where $\psi_{b}(c)=c / b$ is a continuous bijective function in the variable $c$ by Equation (14) and Lemma 3. On the other hand, the right shift mapping

$$
R_{b} u:=u b
$$

from $G$ into $G$ is continuous and bijective in $u$ (see Definition 1). Moreover, $\psi_{b} \circ R_{b} u=u$ and $R_{b} \circ \psi_{b}(c)=c$ for each fixed $b \in G$ and all $u \in G$ and $c \in G$ by (16). Thus $R_{b}$ and $\psi_{b}$ are open mappings, consequently, $U b$ is open.

Similarly for the equation

$$
b y=c
$$

the unique solution is

$$
y=b \backslash c
$$

by the condition of Equation (1).

Therefore, $y=\theta_{b}(c)$, where $\theta_{b}(c)=b \backslash c$ is a continuous bijective function in $c$ according to Lemma 3 and Equation (13). Next we consider the left shift mapping

$$
L_{b} u=b u
$$

for each fixed $b \in G$ and any $u \in G$. This mapping $L_{b}$ is continuous, since the multiplication on $G$ is continuous. Then $L_{b} \circ \theta_{b}(c)=c$ and $\theta_{b} \circ L_{b} u=u$ for every fixed $b \in G$ and all $u \in G$ and $c \in G$ by Equation (15). Therefore $\theta_{b}$ and $L_{b}$ are open mappings. Thus the subset $b U$ is open in $G$.

Proposition 1. Let $(G, \tau)$ be a topological metagroup and let $U$ and $V$ be subsets in $G$ such that either $U$ or $V$ is open, then UV is open in G.

Proof. In view of Lemma 4 either $U b$ is open in $G$ for each $b \in V$ or $a V$ is open in $G$ for each $a \in U$ respectively, consequently, $U V=\{x=u v: u \in U, v \in V\}=\bigcup_{b \in V} U b=\bigcup_{a \in U} a V$ is open in $G$.

Theorem 1. If $G$ is a $T_{1}$ topological metagroup and $\mathcal{C}_{0}$ is a closed subgroup in a center $\mathcal{C}(G)$ such that $t(a, b, c) \in \mathcal{C}_{0}$ for each $a, b$ and $c$ in $G$, then its quotient $G / \mathcal{C}_{0}$ is a $T_{1} \cap T_{3.5}$ topological group.

Proof. Let $\tau$ be a $T_{1}$ topology on $G$ relative to which $G$ is a topological metagroup. Then each point $x$ in $G$ is closed, since $G$ is the $T_{1}$ topological space (see Section 1.5 in [29]). From the continuity of multiplication and the left inversion and right inversion it follows that the center $\mathcal{C}(G)$ is closed in $G$. Therefore the subgroup $\mathcal{C}_{0}$ is closed in $G$. As is tradition, the notation:

$$
\begin{aligned}
& A B=\{x=a b: a \in A, b \in B\} \\
& \operatorname{Inv}_{l}(A)=\{x=a \backslash e: a \in A\} \\
& \operatorname{Inv}_{r}(A)=\{x=e / a: a \in A\}
\end{aligned}
$$


is used for subsets $A$ and $B$ in $G$. Then from Equations (4)-(8) it follows that for each $a, b, c$ in $G$ the identities take place.

$\left(\left(a \mathcal{C}_{0}\right)\left(b \mathcal{C}_{0}\right)\right)\left(c \mathcal{C}_{0}\right)=\left(a \mathcal{C}_{0}\right)\left(\left(b \mathcal{C}_{0}\right)\left(c \mathcal{C}_{0}\right)\right)$ and $a \mathcal{C}_{0}=\mathcal{C}_{0} a$. Evidently $e \mathcal{C}_{0}=\mathcal{C}_{0}$. In view of Lemmas 1 and $2\left(a \mathcal{C}_{0}\right) \backslash e=e /\left(a \mathcal{C}_{0}\right)$, consequently, for each $a \mathcal{C}_{0} \in G / \mathcal{C}_{0}$ a unique inverse $\left(a \mathcal{C}_{0}\right)^{-1}$ exists. Thus the quotient $G / \mathcal{C}_{0}$ of $G$ by $\mathcal{C}_{0}$ is a group. Since the topology $\tau$ on $G$ is $T_{1}$ and $\mathcal{C}_{0}$ is closed in $G$, then the quotient topology $\tau_{q}$ on $G / \mathcal{C}_{0}$ is also $T_{1}$. By virtue of Theorem 8.4 in [30] this implies that $\tau_{q}$ is a $T_{1} \cap T_{3.5}$ topology on $G / \mathcal{C}_{0}$.

We remind reader of the following.

Definition 2. Let $G$ be a topological metagroup. For a subset $U$ in $G$ it is put:

$$
\begin{aligned}
& \mathcal{L}_{U, G}:=\{(x, y) \in G \times G: x \backslash y \in U\} \\
& \mathcal{R}_{U, G}:=\{(x, y) \in G \times G: y / x \in U\}
\end{aligned}
$$

The family of all subsets $\mathcal{L}_{U, G}$ (or $\mathcal{R}_{U, G}$ ) with $U$ being an open neighborhood of e will be called a left (right correspondingly) uniform structure on $G$ and denoted by $\mathcal{L}_{G}$ (respectively $\mathcal{R}_{G}$ ).

Theorem 2. Assume that $G$ is a $T_{1}$ topological metagroup and a function $t$ on $G$ is defined by Equation (10). Then for each compact subset $S$ in $G$ and each open neighborhood $V$ of e there exists an open symmetric neighborhood $U$ of $e$ in $G$ such that

$$
t\left(\left(u_{1} a\right) v_{1},\left(u_{2} b\right) v_{2},\left(u_{3} c\right) v_{3}\right) / t(a, b, c) \in V
$$

for every $a, b, c$ in $S$ and $u_{j}, v_{j}$ in $U$ for each $j \in\{1,2,3\}$.

Proof. Let $\theta: G \rightarrow G / \mathcal{C}$ denote the quotient map of $G$ on the quotient group $G / \mathcal{C}=: Q$ provided by Theorem 1, where $\mathcal{C}=\mathcal{C}(G)$. That is $a \mathcal{C}=\theta(a)$ for each $a \in G$. From Lemma 2 it follows that $t$ on $G$ induces a continuous map $\hat{t}$ on $Q$ with values in $\mathcal{C}$, where $\hat{t}(\theta(a), \theta(b), \theta(c))=t(a, b, c)$ for each $a, b, c$ in $G$.

Since $S$ is compact in $G$ and $\theta$ is continuous from $(G, \tau)$ into $\left(Q, \tau_{q}\right)$, then $\theta(S)=: \hat{S}$ is compact in $Q$ by Theorem 3.1.10 in [29]. Therefore,

$$
\hat{t}(\hat{S}, \hat{S}, \hat{S}):=\{y=\hat{t}(\hat{a}, \hat{b}, \hat{c}): \hat{a} \in \hat{S}, \hat{b} \in \hat{S}, \hat{c} \in \hat{S}\}
$$

is a compact subset in $\mathcal{C}$, where $\mathcal{C}$ is supplied with the topology inherited from $G$. In view of Proposition 1 if $U$ is an open symmetric neighborhood of $e$ in $G$, then $\hat{U}=\theta(U)$ is an open symmetric neighborhood of the unit element $e_{Q}$ in $Q$. The group $\mathcal{C}$ is commutative, hence Equation (32) is equivalent to

$$
\hat{t}\left(\hat{u}_{1} \hat{a} \hat{v}_{1}, \hat{u}_{2} \hat{b} \hat{v}_{2}, \hat{u}_{3} \hat{c} \hat{v}_{3}\right) \in \hat{t}(\hat{a}, \hat{b}, \hat{c})(V \cap \mathcal{C})
$$

for every $\hat{a}, \hat{b}, \hat{c}$ in $\hat{S}$ and $\hat{u}_{j}, \hat{v}_{j}$ in $\hat{U}$ for each $j \in\{1,2,3\}$, where $\hat{a}=\theta(a)$ for each $a \in G$.

Naturally $Q^{\otimes 3}=Q \otimes Q \otimes Q$ is a topological group as the direct product of three copies of the topological group $Q$ (see Theorem 6.2 in [30]). Since $\hat{S} \times \hat{S} \times \hat{S}$ is compact in the topological group $Q^{\otimes 3}$, then $\hat{t}$ is uniformly continuous on $\hat{S} \times \hat{S} \times \hat{S}$ relative to the uniformities $\mathcal{L}_{Q^{\otimes 3}}$ and $\mathcal{R}_{Q^{\otimes 3}}$; consequently, there exists an open symmetric neighborhood $W$ of $e_{Q}$ in $Q$ such that Equation (33) is satisfied for every $\hat{a}, \hat{b}, \hat{c}$ in $\hat{S}$ and $\hat{u}_{j}, \hat{v}_{j}$ in $W$ for each $j \in\{1,2,3\}$. Take an open symmetric neighborhood $U$ of $e$ in $G$ such that $U \subseteq \theta^{-1}(W)$. With this $U$ Equation (32) is fulfilled.

Proposition 2. Suppose that $(G, \tau)$ is a topological metagroup, $S$ is a compact subset in $G, q$ is a fixed element in $G, V$ is an open neighborhood of the unit element $e$. Then there are elements $b_{1}, \ldots, b_{m}$ in $G$ and an open symmetric neighborhood $U$ of e such that

$$
U \subset V
$$


and

$$
\left\{b_{1} \backslash(q U), \ldots, b_{m} \backslash(q U)\right\}
$$

is an open covering of $S$, and

$$
b_{k}\left(b_{k} \backslash(q U)\right) \subset q V
$$

for each $k=1, \ldots, m$.

Proof. Since the left $I n v_{l}$ and right $I n v_{r}$ inversions are homeomorphisms of $G$ onto itself as the topological space, then for each open neighborhood $W$ of $e$ the intersection

$$
W \cap \operatorname{Inv}_{l}(W) \cap \operatorname{Inv}_{r}(W)
$$

is an open symmetric neighborhood of $e$, since $\operatorname{Inv} v_{l} \circ \operatorname{Inv} v_{r}(b)=b$ and $\operatorname{In} v_{r} \circ \operatorname{In} v_{l}(b)=b$ for each $b$ in $G$. The multiplication is continuous on $G$, hence the left shift mapping $L_{b}(x)=b x$ is continuous on $G$ in the variable $x$. On the other hand, the left inversion $I n v_{l}$ is continuous on $G$.

In view of Lemmas 1, 4 and the compactness of $S$ for each open symmetric neighborhood $U$ of $e$ in $G$ there are $b_{1}, \ldots, b_{m}$ in $G$ such that $\left\{b_{1} \backslash(q U), \ldots, b_{m} \backslash(q U)\right\}$ is an open covering of $S$. Then Equation (15) and inclusion (34) imply inclusion (36).

Corollary 1. Let $G$ be a topological metagroup. Then for each open neighborhood $W$ of $e$ in $G$ there exists an open symmetric neighborhood $U$ of e such that

$$
(\forall x \forall y((x \in G) \&(y \in G) \&(x \backslash y \in U))) \Rightarrow(y \in x W)
$$

and

$$
(\forall x \forall y((x \in G) \&(y \in G) \&(y / x \in U))) \Rightarrow(y \in W x)
$$

Proof. This follows from property (37), Equations (15) and (16).

Theorem 3. Let $G$ and $H$ be topological metagroups and let $f: G \rightarrow H$ be a continuous map so that for each open neighborhood $V$ of a unit element $e_{H}$ in $H$ a compact subset $K_{V}$ in $G$ exists such that $f\left(G-K_{V}\right) \subset V$. Then $f$ is uniformly $\left(\mathcal{L}_{G}, \mathcal{L}_{H}\right)$ continuous and uniformly $\left(\mathcal{R}_{G}, \mathcal{R}_{H}\right)$ continuous.

Proof. Since each open neighborhood of $e_{H}$ in $H$ contains an open symmetric neighborhood according to property (37), then it suffices to consider an open symmetric neighborhood $V$ of $e_{H}$ in $H$. Let $V_{1}$ be an open symmetric neighborhood of $e_{H}$ in $H$ such that $V_{1}^{2} \subset V$, where $A B=\{c \in H: c=a b$, $a \in A, b \in B\}, A^{2}=A A$ for subsets $A$ and $B$ in $H$. By the conditions of this theorem, a compact subset $K_{V_{1}}$ in $G$ exists such that $f\left(G-K_{V_{1}}\right) \subset V_{1}$.

For a subset $A$ of the metagroup $G$ let

$$
P(A)=\left(P_{0}(A) \cup\{e\}\right)\left(P_{0}(A) \cup\{e\}\right)
$$

where $P_{0}(A)=A \cup \operatorname{Inv}_{l}(A) \cup \operatorname{Inv}_{r}(A)$, hence $A \subset P_{0}(A)$ and $P_{0}(A) \cup\{e\} \subset P(A)$. Then $S_{1}=P\left(K_{V_{1}}\right)$ is a compact subset in $G$, since the left $I n v_{l}$ and right $I n v_{r}$ inversions and multiplication are continuous on $G$ (see Theorems 3.1.10, 8.3.13-8.3.15 in [29]), hence $R_{1}=P\left(f\left(S_{1}\right)\right)$ is compact in $H$. 
Since the multiplication in $H$ is continuous, then for each open neighborhood $Y$ of $e_{H}$ there exists an open neighborhood $X$ of $e_{H}$ such that $X^{2} \subset Y$. By virtue of Theorem 2 there exists an open symmetric neighborhood $V_{2}$ of $e_{H}$ in $H$ such that

$$
\left[t_{H}\left(\left(V_{2} a\right) V_{2},\left(V_{2} b\right) V_{2},\left(V_{2} c\right) V_{2}\right) V_{2} / t_{H}(a, b, c)\right]^{2} \subset V_{1}
$$

for every $a, b, c$ in $R_{1}$. For $V_{2}$ there exists a compact subset $K_{V_{2}}$ in $G$ such that $f\left(G-K_{V_{2}}\right) \subset V_{2}$ by the conditions of this theorem. If $A$ and $B$ are compact subsets in $G$, then their union $A \cup B$ is also compact. Therefore it is possible to choose $K_{V_{2}}$ such that $K_{V_{1}} \subset K_{V_{2}}$, since $V_{2} \subset V_{1}$ and $(G-A)-B=G-(A \cup B) \subset G-A$. We take $S_{2}=P\left(K_{V_{2}}\right)$ by Equation (40), consequently, $S_{1} \subset S_{2}$, since $K_{V_{1}} \subset K_{V_{2}}$.

From the continuity of the map $f$ and Lemma 4 it follows that for each $x \in G$ open symmetric neighborhoods $W_{x, l}$ and $W_{x, r}$ of $e$ in $G$ exist such that

$$
\begin{aligned}
f\left(x W_{x, l}^{2}\right) \subset\left(f(x) V_{2}\right) \text { and } & f\left(W_{x, r}^{2} x\right) \subset\left(V_{2} f(x)\right) \text {; consequently, } \\
& f\left(x W_{x}^{2}\right) \subset\left(f(x) V_{2}\right), f\left(W_{x}^{2} x\right) \subset\left(V_{2} f(x)\right)
\end{aligned}
$$

for an open symmetric neighborhood $W_{x}=W_{x, l} \cap W_{x, r}$ of $e$ in G. The compactness of $S_{2}$ implies that coverings $\left\{x W_{x}: x \in S_{2}\right\}$ and $\left\{W_{y} y: y \in S_{2}\right\}$ of $S_{2}$ have finite subcoverings $\left\{x_{j} W_{x_{j}}: x_{j} \in S_{2}\right.$, $j=1, \ldots, n\}$ and $\left\{W_{y_{i}} y_{i}: y_{i} \in S_{2}, i=1, \ldots, m\right\}$. Hence

$$
W=\bigcap_{j=1}^{n} W_{x_{j}} \cap \bigcap_{i=1}^{m} W_{y_{i}}
$$

is a symmetric open neighborhood of $e$ in G. Therefore, according to Theorem 2, there exists an open symmetric neighborhood $U$ of the unit element $e$ in $G$ such that

$$
\left[t_{G}((U a) U,(U b) U,(U c) U) U / t_{G}(a, b, c)\right]^{2} \subset W_{0}
$$

for every $a, b, c$ in $S_{2}$, where $W_{0}$ is an open symmetric neighborhood of $e$ in $G$ such that $W_{0}^{2} \subset W$.

Let $x$ and $y$ in $G$ be such that $x \backslash y \in U$. Then Equation (15) implies that

$$
y \in x U
$$

There are several options. Consider first the case $x \in K_{V_{2}}$. From Equation (43), Inclusions (44), (45) and Corollary 1 it follows that there exists $j \in\{1, \ldots, n\}$ such that $x \in x_{j} W_{x_{j}}$ and $y \in x_{j} W_{x_{j}}^{2}$. Therefore, Inclusions (41) and (42) imply that $f(x) \backslash f(y) \in V$.

From $x \backslash y \in U$ and Equation (15) it follows that $y=x u$ for a unique $u \in U$. Hence

$$
x=y(e / u) t(e / u, u, u \backslash e) / t(y / u, u, u \backslash e)
$$

according to Equations (14) and (16).

If $y \in K_{V_{2}}$, then similarly from Equations (43), (46), inclusions (44), (45) and Corollary 1 it follows that there exists $k \in\{1, \ldots, n\}$ such that $y \in x_{k} W_{x_{k}}$ and $x \in x_{k} W_{x_{k}}^{2}$, since an open neighborhood $U$ of $e$ is symmetric and $t(a, b, e)=t(a, e, b)=t(e, a, b)=e$ for each $a$ and $b$ in $G$. Therefore, $f(x) \backslash f(y) \in V$ by Inclusions (41) and (42), since the considered neighborhoods of $e_{H}$ in $H$ and $e=e_{G}$ in $G$ are symmetric and $S_{2}=P\left(K_{V_{2}}\right)$ (see Equation $(40)$ ).

It remains the case $x \in G-K_{V_{2}}$ and $y \in G-K_{V_{2}}$. Therefore, $f(x) \in V_{2}$ and $f(y) \in V_{2}$. According to the choice of $R_{1}$ we have $e_{H} \in R_{1}$. The open neighborhoods $V, V_{1}, V_{2}$ of $e_{H}$ in $H$ are symmetric. From Inclusion (41), Equation (15) and the inclusion $V_{1}^{2} \subset V$, it follows that $f(x) \backslash f(y) \in V$. Taking into account the inclusion $K_{V_{1}} \subset K_{V_{2}}$ we get that $f$ is uniformly $\left(\mathcal{L}_{G}, \mathcal{L}_{H}\right)$ continuous. 
The uniform $\left(\mathcal{R}_{G}, \mathcal{R}_{H}\right)$ continuity is proved analogously using the finite subcovering $\left\{W_{y_{i}} y_{i}: y_{i} \in S_{2}, i=1, \ldots, m\right\}$ and Corollary 1 .

Corollary 2. Let $G$ be a locally compact metagroup, let $f \in C_{0}(G)$ and let $H=(\mathbf{C},+)$ be the complex field $\mathbf{C}$ considered as an additive group. Then $f$ is uniformly $\left(\mathcal{L}_{G}, \mathcal{L}_{H}\right)$ continuous and uniformly $\left(\mathcal{R}_{G}, \mathcal{R}_{H}\right)$ continuous.

This corollary follows from Theorem 3 as its particular case.

\section{Products of Topological Metagroups}

In this section, we demonstrate that there are abundant families of topological metagroups besides those described in the introduction.

Theorem 4. Let $\left(G_{j}, \tau_{j}\right)$ be a family of topological metagroups (see Definition 1), where $j \in J, J$ is a set. Then their direct product $G=\prod_{j \in J} G_{j}$ relative to the Tychonoff product topology $\tau$ is a topological metagroup and

$$
\mathcal{C}(G)=\prod_{j \in J} \mathcal{C}\left(G_{j}\right)
$$

Proof. The direct product of topological loops is a topological loop (see [2,3]). Thus Conditions (1)-(3) are satisfied.

Each element $a \in G$ is written as $a=\left\{a_{j}: \forall j \in J, a_{j} \in G_{j}\right\}$. From Equations(4)-(7) we infer that

$$
\begin{gathered}
\operatorname{Com}(G):=\{a \in G: \forall b \in G, a b=b a\}=\left\{a \in G: a=\left\{a_{j}: \forall j \in J, a_{j} \in G_{j}\right\} ; \forall b \in G,\right. \\
\left.b=\left\{b_{j}: \forall j \in J, b_{j} \in G_{j}\right\} ; \forall j \in J, a_{j} b_{j}=b_{j} a_{j}\right\}=\prod_{j \in J} \operatorname{Com}\left(G_{j}\right) \\
N_{l}(G):=\{a \in G: \forall b \in G, \forall c \in G,(a b) c=a(b c)\}=\left\{a \in G: a=\left\{a_{j}: \forall j \in J, a_{j} \in G_{j}\right\} ; \forall b \in G\right. \\
\left.b=\left\{b_{j}: \forall j \in J, b_{j} \in G_{j}\right\} ; \forall c \in G, c=\left\{c_{j}: \forall j \in J, c_{j} \in G_{j}\right\} ; \forall j \in J,\left(a_{j} b_{j}\right) c_{j}=a_{j}\left(b_{j} c_{j}\right)\right\}=\prod_{j \in J} N_{l}\left(G_{j}\right)
\end{gathered}
$$

and similarly

$$
N_{m}(G)=\prod_{j \in J} N_{m}\left(G_{j}\right)
$$

and

$$
N_{r}(G)=\prod_{j \in J} N_{r}\left(G_{j}\right)
$$

This and Equation (8) imply that

$$
N(G)=\prod_{j \in J} N\left(G_{j}\right)
$$

Thus

$$
\mathcal{C}(G):=\operatorname{Com}(G) \cap N(G)=\prod_{j \in J} \mathcal{C}\left(G_{j}\right)
$$

Let $a, b$ and $c$ be in $G$, then

$$
\begin{gathered}
(a b) c=\left\{\left(a_{j} b_{j}\right) c_{j}: \forall j \in J, a_{j} \in G_{j}, b_{j} \in G_{j}, c_{j} \in G_{j}\right\}=\left\{t_{G_{j}}\left(a_{j}, b_{j}, c_{j}\right) a_{j}\left(b_{j} c_{j}\right):\right. \\
\left.\forall j \in J, a_{j} \in G_{j}, b_{j} \in G_{j}, c_{j} \in G_{j}\right\}=t_{G}(a, b, c) a(b c)
\end{gathered}
$$

where

$t_{G}(a, b, c)=\left\{t_{G_{j}}\left(a_{j}, b_{j}, c_{j}\right): \forall j \in J, a_{j} \in G_{j}, b_{j} \in G_{j}, c_{j} \in G_{j}\right\}$.

Therefore, Equations (53) and (54) imply that Condition (10) also is satisfied. Thus $G$ is a topological metagroup. 
Remark 1. Let $A$ and $B$ be metagroups and let $\mathcal{C}$ be a commutative group such that

$$
\mathcal{C}_{m}(A) \hookrightarrow \mathcal{C}, \mathcal{C}_{m}(B) \hookrightarrow \mathcal{C}, \mathcal{C} \hookrightarrow \mathcal{C}(A) \text { and } \mathcal{C} \hookrightarrow \mathcal{C}(B)
$$

where $\mathcal{C}_{m}(A)$ denotes a minimal subgroup in $\mathcal{C}(A)$ containing $t_{A}(a, b, c)$ for every $a, b$ and $c$ in $A$.

Using direct products it is always possible to extend either $A$ or $B$ to get such a case. In particular, either $A$ or $B$ may be a group. On $A \times B$ an equivalence relation $\Xi$ is considered such that

$$
(\gamma v, b) \Xi(v, \gamma b) \text { and }(\gamma v, b) \Xi \gamma(v, b) \text { and }(\gamma v, b) \Xi(v, b) \gamma
$$

for every $v$ in $A, b$ in $B$ and $\gamma$ in $\mathcal{C}$.

$$
\text { Let } \phi: A \rightarrow \mathcal{A}(B) \text { be a single-valued mapping, }
$$

where $\mathcal{A}(B)$ denotes a family of all bijective surjective single-valued mappings of $B$ onto $B$ subjected to the conditions given below. If $a \in A$ and $b \in B$, then it will be written shortly $b^{a}$ instead of $\phi(a) b$, where $\phi(a): B \rightarrow B$. Let also

$$
\begin{aligned}
& \eta_{\phi}: A \times A \times B \rightarrow \mathcal{C}, \kappa_{\phi}: A \times B \times B \rightarrow \mathcal{C} \\
& \text { and } \xi_{\phi}:((A \times B) / \Xi) \times((A \times B) / \Xi) \rightarrow \mathcal{C}
\end{aligned}
$$

be single-valued mappings written shortly as $\eta, \kappa$ and $\xi$ correspondingly such that

$$
\begin{gathered}
\left(b^{u}\right)^{v}=b^{v u} \eta(v, u, b), e^{u}=e, b^{e}=b ; \\
\eta(v, u, \gamma b)=\eta(v, u, b) ; \\
(c b)^{u}=c^{u} b^{u} \kappa(u, c, b) ;
\end{gathered}
$$

$\kappa(u, \gamma c, b)=\kappa(u, c, \gamma b)=\kappa(u, c, b)$ and

$$
\begin{gathered}
\kappa(u, \gamma, b)=\kappa(u, b, \gamma)=e ; \\
\xi((\gamma u, c),(v, b))=\xi((u, c),(\gamma v, b))=\xi((u, c),(v, b))
\end{gathered}
$$

and $\xi((\gamma, e),(v, b))=e$ and $\xi((u, c),(\gamma, e))=e$ for every $u$ and $v$ in $A, b$ and $c$ in $B, \gamma$ in $\mathcal{C}$, where $e$ denotes the neutral element in $\mathcal{C}$ and in $A$ and $B$.

We put

$$
\left(a_{1}, b_{1}\right)\left(a_{2}, b_{2}\right)=\left(a_{1} a_{2}, \xi\left(\left(a_{1}, b_{1}\right),\left(a_{2}, b_{2}\right)\right) b_{1} b_{2}^{a_{1}}\right)
$$

for each $a_{1}, a_{2}$ in $A, b_{1}$ and $b_{2}$ in $B$.

The Cartesian product $A \times B$ supplied with such a binary operation (63) will be denoted by $A \otimes \otimes^{\phi, \eta, \kappa, \xi} B$.

Theorem 5. Let the conditions of Remark 1 be satisfied. Then the Cartesian product $A \times B$ supplied with a binary operation of Equation (63) is a metagroup.

For the proof of this theorem see Theorem 9 in [27].

Definition 3. The metagroup $A \otimes^{\phi, \eta, \kappa, \xi} B$ of Theorem 5 is called a smashed product of metagroups $A$ and $B$ with smashing factors $\phi, \eta, \kappa$ and $\xi$.

Particularly, it is possible to consider the topological metagroup fulfilling the condition: there exists a compact subgroup $\mathcal{C}_{0}=\mathcal{C}_{0}(G)$ in $\mathcal{C}(G)$ such that

$$
t_{G}(a, b, c) \in \mathcal{C}_{0} \text { for every } a, b \text { and } c \text { in } G
$$


Corollary 3. (i). Assume that conditions of Theorem 4 are satisfied and for each $j \in J$ a metagroup $G_{j}$ satisfies Condition (64). Then the product metagroup $G$ satisfies Condition (64).

(ii). Moreover, if $G_{j}$ is compact for all $j \in J_{0}$ and locally compact for each $j \in J \backslash J_{0}$, where $J_{0} \subset J$ and $J \backslash J_{0}$ is a finite set, then $G$ is locally compact.

Proof. (i). Using Equation (54) it is sufficient to take $\mathcal{C}_{0}(G)=\prod_{j \in J} \mathcal{C}_{0}\left(G_{j}\right)$, since the direct product of compact commutative groups $\mathcal{C}_{0}\left(G_{j}\right)$ is a compact commutative group $\mathcal{C}_{0}(G)$ (see [29,30]).

The last assertion (ii) follows from the known fact that $G$ as a topological space is locally compact under the imposed above conditions by Theorem 3.3.13 in [29].

Corollary 4. Suppose that the conditions of Remark 1 are fulfilled and $A$ and $B$ are topological metagroups and smashing factors $\phi, \eta, \kappa, \xi$ are jointly continuous by their variables. Suppose also that $A \otimes^{\phi, \eta, \kappa, \xi} B$ is supplied with a topology induced from the Tychonoff product topology on $A \times B$. Then $A \otimes^{\phi, \eta, \kappa, \xi} B$ is a topological metagroup.

Corollary 5. If the conditions of Corollary 4 are satisfied and metagroups $A$ and B are locally compact, then $A \otimes^{\phi, \eta, \kappa, \xi} B$ is locally compact. Moreover, if $A$ and $B$ satisfy Condition (64) and ranges of $\eta, \kappa, \xi$ are contained in $\mathcal{C}_{0}(A) \mathcal{C}_{0}(B)$, then $A \otimes^{\phi, \eta, \kappa, \xi} B$ satisfies Condition (64).

Proof. Corollaries 4 and 5 follow from Theorems 2.3.11, 3.2.4, 3.3.13 in [29], Lemma 3 and Theorems 1, 2,5 , since

$\mathcal{C}_{0}(A) \mathcal{C}_{0}(B) \subseteq \mathcal{C} \subseteq \mathcal{C}(A) \cap \mathcal{C}(B)$ and $\mathcal{C}_{0}(A) \mathcal{C}_{0}(B)$ is a compact subgroup in $A \otimes^{\phi, \eta, \kappa, \xi} B$.

Example 1. Assume that $X$ is a $T_{1} \cap T_{3.5}$ topological space, $G$ is a $T_{1} \cap T_{3.5}$ topological metagroup (not a group). We consider the space $C(X, G)$ of all continuous maps $f: X \rightarrow G$ supplied with the compact-open topology and the point-wise multiplication $(f g)(x)=f(x) g(x)$ for each $f, g$ in $C(X, G)$ and $x \in X$. Then this constriction and Definition 1 imply that there exist $(f \backslash g)(x)=f(x) \backslash g(x),(f / g)(x)=f(x) / g(x), e_{C(X, G)}(x)=e_{G}$ and $t_{C(X, G)}(f, g, h)(x)=t_{G}(f(x), g(x), h(x))$ for each $f, g$ and $h$ in $C(X, G)$ and $x \in X$. It can be easily seen that $C(X, G)$ is the topological metagroup (not a group). Generally $C(X, G)$ may be nonlocally compact metagroup for nontrivial $X$ and $G$ with the small inductive dimension ind $(X) \leq$ ind $(G)$ and the topological weight $w(X) \geq \aleph_{0}, w(G)>7$.

Other metagroups are given in Examples 1-3 in [28]. In general they are nonassociative and hence different from groups.

Example 2. Particularly, $H_{r}:=\left\{i_{j},-i_{j}, i_{j} \mathbf{i},-i_{j} \mathbf{i}: j \in \Lambda_{r}\right\}$ is the metagroup, where either $\Lambda_{r}=\left\{0, \ldots, 2^{r}-1\right\}$ with $3 \leq r \in \mathbf{N}$ or $\Lambda_{\infty}=\{j: 0 \leq j \in \mathbf{Z}\}, i_{j}$ and $i_{j} \mathbf{i}$ with $j \in \Lambda$ are the canonical generators of the complexified Cayley-Dickson algebra $\mathcal{A}_{r, \mathbf{C}}$ such that $i_{j} \mathbf{i}=\mathbf{i} i_{j}$ and $\mathbf{i}^{2}=-1$; where $i_{j}$ with $j \in \Lambda_{r}$ are the canonical generators of the Cayley-Dickson algebra $\mathcal{A}_{r, \mathbf{R}}$ over the real field $\mathbf{R}, i_{0}=1$ (for more details see Example 1 in [28]). Therefore, relative to the discrete topology $H_{r}$ is the compact nonassociative metagroup for each $3 \leq r \in \mathbf{N}$; while $H_{\infty}$ is the locally compact nonassociative metagroup and $H_{\infty}$ is not compact. Then we put

$$
H_{\alpha, \mu, p}:=\prod_{j \in \alpha, \mu(j) \in \Omega_{p}} H_{\mu(j)}
$$

where $\alpha$ is a set, $\Omega_{p}=\{r \in \mathbf{N}: 3 \leq r \leq p\}, 3 \leq p \leq \infty, \mu: \alpha \rightarrow \Omega_{p}$ is a map. We supply $H_{\alpha, \mu, p}$ with the Tychonoff product topology, hence $H_{\alpha, \mu, p}$ is the topological nonassociative metagroup by Theorem 4 . If $\operatorname{card}(\alpha) \geq \aleph_{0}$, then $H_{\alpha, u, p}$ is not discrete. For each $3 \leq p \in \mathbf{N}$, each set $\alpha$ and every map $\mu: \alpha \rightarrow \Omega_{p}$, the metagroup $H_{\alpha, \mu, p}$ is compact. If $1 \leq \operatorname{card}\{j: \mu(j)=\infty\}<\aleph_{0}$, then $H_{\alpha, \mu, \infty}$ is locally compact and noncompact by Corollary 3. For each $\alpha$ and $\mu$ such that card $\{j: \mu(j)=\infty\} \geq \aleph_{0}$ the metagroup $H_{\alpha, \mu, \infty}$ is not locally compact. 
Example 3. If $A$ and $B$ are topological metagroups and $A$ has the positive small inductive dimension ind $(A)>0$, then ind $(A \times B) \geq \operatorname{ind}(A)>0$ according to Theorems 7.1.1 in [29] and 4 above. In particular, $A$ may be a group and $B$ be the nonassociative metagroup, hence $A \times B$ is the nonassociative topological metagroup with ind $(A \times B)>0$.

Example 4. Other examples follow from Remark 3 and Theorem 9 in [27], Remark 4 and Theorem 6 in [28], Examples 1-3, Theorem 5 and Corollaries 4 and 5 above providing nonassociative topological metagroups $A \otimes^{\phi, \eta, \kappa, \xi} B$, which may be either locally compact or nonlocally compact or (and) with ind $\left(A \otimes^{\phi, \eta, \kappa, \xi} B\right)>0$.

Remark 2. From Theorems 4 and 5 and Corollaries 3-5, it follows that taking nontrivial $\eta, \kappa$, and $\xi$ and starting even from groups with nontrivial $\mathcal{C}\left(G_{j}\right)$ or $\mathcal{C}(A)$ it is possible to construct new metagroups with nontrivial $\mathcal{C}_{0}(G)$ and ranges $t_{G}(G, G, G)$ of $t_{G}$ may be infinite and nondiscrete. With suitable smashing factors $\phi, \eta, \kappa$ and $\xi$ and with nontrivial metagroups or groups $A$ and $B$ it is easy to get examples of metagroups in which $e / a \neq a \backslash$ e for an infinite family of elements $a$ in $A \otimes^{\phi, \eta, \kappa, \xi} B$. The latter and Examples 1-3 above show that there are a lot of metagroups $G$ for which $t_{G}(a, b, c)$ depend nontrivially on all arguments $a, b$, and $c$ in $G$.

In Theorem 5, instead of the family of all automorphisms of $B$, we used the family $\mathcal{A}(B)$ (see Remark 1). In particular, $\phi(a) \in \mathcal{A}(B)$ may be different from the automorphism of $B$ for some $a \in A$. Moreover multipliers $t_{G}$ in Definition $1, \xi, \eta, \kappa$ in Remark 1 and Theorem 5 provide left $L_{q}$ or right $R_{q}$ shifts on $G$ or $A$, or $B$ respectively, where

$q \in\left\{t_{G}(a, b, c) ; \xi\left(\left(a_{1}, b_{1}\right),\left(a_{2}, b_{2}\right)\right) ; \eta\left(a_{1}, a_{2}, b_{1}\right) ; \kappa\left(a_{1}, b_{1}, b_{2}\right)\right\}$

with $a, b$ and $c$ in $G, a_{1}$ and $a_{2}$ in $A ; b_{1}$ and $b_{2}$ in $B$; where $L_{q} a_{1}=q a_{1}$ for each $q \in A$ and $a_{1} \in A$, $R_{p} b_{1}=b_{1} p$ for each $p \in B$ and $b_{1} \in B$, etc. The latter means that the maps $L_{q}$ and $R_{q}$ are bijective (injective and surjective). Then $L_{q}$ and $R_{q}$ are different from automorphisms of $G, A, B$ and of $A \otimes^{\phi, \eta, \kappa, \xi} B$ for each $q \neq e$, since each automorphism $\psi$ of $G$ satisfies the restrictions $\psi(a b)=\psi(a) \psi(b)$, $\psi(a \backslash b)=\psi(a) \backslash \psi(b), \psi(a / b)=\psi(a) / \psi(b)$ for each $a$ and $b$ in $G$. Moreover, in the smashed twisted wreath product of metagroup construction operators $\hat{S}_{d}, T_{h}, L_{w_{j}}\left(d, d_{1}, v\right)$ on $F$ are used which are generally not automorphisms of $F$ (see Theorem 6 in [28]). This implies that the class of metagroups is different from the class of gyrogroups.

Example 2 means that $\mathcal{A}_{3, \mathrm{C}}$ is the complexified octonion algebra $\mathbf{O}_{\mathrm{C}}$ and $\mathcal{A}_{3, \mathbf{R}}$ is the octonion algebra $\mathbf{O}$ over $\mathbf{R}$. Thus the Cayley-Dickson algebras are metagroup algebras. The complexified octonion algebra $\mathbf{O}_{\mathbf{C}}$ was used, for example, in physics and geometry in [16,31,32]. The metagroup algebra $\mathcal{A}_{r, \mathbf{C}}$ was used in $[12,18,19,25]$ for solutions of PDEs.

\section{Conclusions}

The results of this article can be used for further studies of measures on homogeneous spaces and noncommutative manifolds related with metagroups. They can be used in topological algebra, harmonic analysis on nonassociative algebras and metagroups and loops, representation theory, geometry, mathematical physics, quantum field theory, particle physics and PDEs, etc. Besides them it is interesting to mention possible applications in mathematical coding theory, analysis of information flows and distributed databases [33-36], because codes and databases are based on topological-algebraic binary systems. Other very important applications include harmonic analysis, mathematical physics, quantum field theory, gauge theory, quantum gravity, representation theory of metagroups and nonassociative algebras, and noncommutative geometry $[16,20-22,26,30,37,38]$.

Funding: This research received no external funding.

Conflicts of Interest: The author declares no conflict of interest. 


\section{References}

1. Bourbaki, N. Algebra; Springer: Berlin, Germany, 1989.

2. Bruck, R.H. A Survey of Binary Systems; Springer: Berlin, Germany, 1971.

3. Kakkar, V. Boolean loops with compact left inner mapping groups are profinite. Topol. Appl. 2018, 244, 51-54. [CrossRef]

4. Pickert, G. Projektive Ebenen; Springer: Berlin, Geramny, 1955.

5. Razmyslov, Y.P. Identities of Algebras and Their Representations; Series Modern Algebra; Nauka: Moscow, Russia, 1989; Volume 14.

6. Vojtěchoivský, P. Bol loops and Bruch loops of order pq up to isotopism. Finite Fields Appl. 2018, 52, 1-9.

7. Ungar, A.A. Midpoints in gyrogroups. Found. Phys. 1996, 26, 1277-1328. [CrossRef]

8. Allcock, D. Reflection groups and octave hyperbolic plane. J. Algebra 1998, 213, 467-498. [CrossRef]

9. Baez, J.C. The octonions. Bull. Am.Math. Soc. 2002, 39, 145-205. [CrossRef]

10. Dickson, L.E. The Collected Mathematical Papers; Chelsea Publishing Co.: New York, NY, USA, 1975; Volumes 1-5.

11. Ludkowski, S.V. Automorphisms and derivations of nonassociative $C^{*}$ algebras. Linear Multilinear Algebra 2019, 67, 1531-1538. [CrossRef]

12. Frenod, E.; Ludkowski, S.V. Integral operator approach over octonions to solution of nonlinear PDE. Far East J. Mathem. Sci. (FJMS) 2018, 103, 831-876. [CrossRef]

13. Gilbert, J.E.; Murray, M.A.M. Clifford Algebras and Dirac Operators in Harmonic Analysis. Cambr. Studies in Advanced Mathem; Cambridge University Press: Cambridge, UK, 1991; Volume 26.

14. Girard, P.R. Quaternions, Clifford Algebras And Relativistic Physics; Birkhäuser: Basel, Switzerland, 2007.

15. Gürlebeck, K.; Sprössig, W. Quaternionic And Clifford Calculus for Physicists and Engineers; John Wiley and Sons: Chichester, UK, 1997.

16. Gürsey, F.; Tze, C.-H. On the Role Of Division, Jordan and Related Algebras in Particle Physics; World Scientific Publ. Co.: Singapore, 1996.

17. Kantor, I.L.; Solodovnikov, A.S. Hypercomplex Numbers; Springer: Berlin, Germany, 1989.

18. Ludkowski, S.V. Decompositions of PDE over Cayley-Dickson algebras. Rendic. Dell'Istit. Di Matem. Dell'Univer. Di Trieste. Nuova Ser. 2014, 46, 1-23.

19. Ludkowski, S.V. Integration of vector Sobolev type PDE over octonions. Complex Variab. Elliptic Equat. 2016, 61, 1014-1035.

20. Ludkowski, S.V. Manifolds over Cayley-Dickson algebras and their immersions. Rendic. Dell'Istit. Di Matem. Dell'Univer. Di Trieste. Nuova Ser. 2013, 45, 11-22.

21. Ludkovsky, S.V. Normal families of functions and groups of pseudoconformal diffeomorphisms of quaternion and octonion variables. J. Math. Sci. N. Y. 2008, 150, 2224-2287. [CrossRef]

22. Ludkovsky, S.V. Functions of several Cayley-Dickson variables and manifolds over them. J. Math. Sci. N. Y. 2007, 141, 1299-1330. [CrossRef]

23. Ludkowski, S.V.; Sprössig, W. Ordered representations of normal and super-differential operators in quaternion and octonion Hilbert spaces. Adv. Appl. Clifford Algebr. 2010, 20, 321-342. [CrossRef]

24. Ludkowski, S.V.; Sprössig, W. Spectral theory of super-differential operators of quaternion and octonion variables. Adv. Appl. Clifford Algebr. 2011, 21, 165-191. [CrossRef]

25. Ludkovsky, S.V. Integration of vector hydrodynamical partial differential equations over octonions. Complex Variab. Elliptic Equat. 2013, 58, 579-609. [CrossRef]

26. Castro-Alvaredo, O.A.; Doyon, B.; Fioravanti, D. Conical twist fields and null polygonal Wilson loops. Nuclear Phys. B 2018, 931, 146-178. [CrossRef]

27. Ludkowski, S.V. Cohomology theory of nonassociative algebras. Axioms 2019, 8, 78. [CrossRef]

28. Ludkowski, S.V. Smashed and twisted wreath products of metagroups. Axioms 2019, 8, 127. [CrossRef]

29. Engelking, R. General Topology, 2nd ed.; Sigma Series in Pure Mathematics, V. 6; Heldermann: Berlin, Germany, 1989.

30. Hewitt, E.; Ross, K.A. Abstract Harmonic Analysis; Springer: Berlin, Germany, 1979.

31. Weng, Z.-H. Forces in the complex octonion curved space. Int. J. Geom. Methods Mod. Phys. 2016, 13, 1650076. [CrossRef] 
32. Weng, Z.-H. Some properties of dark matter field in the complex octonion space. Int. J. Mod. Phys. A 2015, 30, 1550212. [CrossRef]

33. Andrianova, E.G.; Raev, V.K.; Filgus, D.I. Determination of the shortest Hamiltonian paths in an arbitrary graph of distributed databases. Russ. Technol. J. 2019, 7, 7-20. [CrossRef]

34. Betten, A. Twisted tensor product codes. Des. Codes Cryptogr. 2008, 47, 191-219. [CrossRef]

35. Blahut, R.E. Algebraic Codes for Data Transmission; Cambridge University Press: Cambridge, UK, 2003.

36. Shum, K.P.; Ren, X.; Wang, Y. Semigroups on semilattice and the constructions of generalized cryptogroups. Southeast Asian Bull. Math. 2014, 38, 719-730.

37. Bogolubov, N.N.; Logunov, A.A.; Oksak, A.I.; Todorov, I.T. General Principles of Quantum Field Theory; Nauka: Moscow, Russia, 1987.

38. Fell, J.M.G.; Doran, R.S. Representations of *-algebras, Locally Compact Groups, and Banach *-algebraic Bundles. V. 1 and V. 2; Academic Press: Boston, MA, USA, 1988.

(C) 2020 by the author. Licensee MDPI, Basel, Switzerland. This article is an open access article distributed under the terms and conditions of the Creative Commons Attribution (CC BY) license (http:// creativecommons.org/licenses/by/4.0/). 